\title{
Reprogramming efficiency of mouse embryonic fibroblasts using pMX-based retroviral expression vectors: testing factors that enhance efficiency.
}

W.W.M. Pim Pijnappel ( $\nabla_{\text {w.pijnappel@erasmusmc.nl ) }}$

Pijnappel/Schöler/Timmers

Daniel Esch

Hans R. Schöler

H.T.Marc Timmers

\section{Method Article}

Keywords: reprogramming, iPS cell, mouse, TFIID, enhancement

Posted Date: March 26th, 2013

DOI: https://doi.org/10.1038/protex.2013.035

License: (c) (1) This work is licensed under a Creative Commons Attribution 4.0 International License. Read Full License 


\section{Abstract}

This protocol describes the testing of factors that can enhance the reprogramming of mouse embryonic fibroblasts $\backslash(\mathrm{MEFs}$ ) to iPC cells using pMX-based retroviral expression.

\section{Procedure}

Note: The success of this experiment is dependent on proper growth of MEFs and high retroviral titers. It is important to take all notes in this protocol into account. Day 1 - evening: plate 293T cells in MEF medium at $2.2 \times 10 \mathrm{e} 6$ cells per $10 \mathrm{~cm}$ dish. 1 dish per transfection. Separate viral supernatants from separate transfections will be combined during infection. Make sure cells are distributed evenly over the plate. Note: for infections, $1 \times 10 \mathrm{ml}$ viral supernatant is sufficient for 40 infections of 250 ul. 1 plate delivers $2 \times 10 \mathrm{ml}$ viral sup for two rounds of infection. Example: $2 \times 10 \mathrm{~cm}$ plates for Oct4-pMX transfection $2 \times 10 \mathrm{~cm}$ plates for Sox2-pMX transfection $2 \times 10 \mathrm{~cm}$ plates for Klf4-pMX transfection $2 \times$ $10 \mathrm{~cm}$ plates for c-Myc-pMX transfection $1 \times 10 \mathrm{~cm}$ plate for each TAF-pMX transfection $\backslash(12$ plates to test TFIID-12). Day 2 - transfect 293T cells from day $1 \backslash$ (cells should look $60-70 \%$ confluent, nicely spread, evenly distributed, without clumps) as follows: o $100 \mu$ DMEM high glucose without additions $\backslash(@ R T) \circ 2$ $\mu \mathrm{g}$ pMX vector containing factor of interest $\cdot$ don't forget to include an empty pMX vector transfection to adjust for additions during infection. o $2 \mu \mathrm{g}$ pClEco packacking plasmid o mix by flicking o $12 \mu \mathrm{l}$ Fugene HD \(@RT) \(Promega cat E2311/E2312) o mix by flicking o 15 min RT o add to cells Day 3 - replace the medium of the transfected 293T cells from day 2 with $10 \mathrm{ml} \mathrm{MEF} \mathrm{medium/10} \mathrm{cm} \mathrm{dish} \mathrm{-} \mathrm{Late} \mathrm{afternoon:}$ start up OG2 MEFs: o plate Passage $\backslash(P) 2$ or 3 of OG2MEFs in MEF medium in $1 \times 15 \mathrm{~cm}$ dish, gelatinized. Plate should be $70-80 \%$ confluent the next morning. Note: the conditions of the MEFs and passage number are critical factors for successful reprogramming. For this reason, they are plated just before the start of the reprogramming experiment to keep the passage number as low as possible and the proliferation rate high. Day 4 - in the early morning, split OG2MEFs at 50,000 cells/well of a gelatinized 6 wells plate $\backslash($ Nalgene). Make sure that the MEFs are evenly distributed over the well. Note: to get an accurate number of healthy OG2 MEFS, it is advised to first plate the cells from a frozen vial and let them adapt for 1 night. Then, the next morning the appropriate number of cells can be plated in 6 wells plates. in the late afternoon, start first infection as follows: o harvest the supernatant of the transfected 293T plates using a syringe and filter sterilize through a $0.45 \mu \mathrm{m}$ low protein binding filter. This is the viral supernatant. o Add fresh MEF medium $\backslash(10 \mathrm{ml} / 10 \mathrm{~cm}$ dish) to the transfected 293T cells to allow a second harvest of viral supernatant after $24 \mathrm{hr}$. o First prepare the infection mix containing all viral combinations in $15 \mathrm{ml}$ tubes - Use $250 \mu \mathrm{l}$ viral supernatant per factor - Add empty pMX viral supernatant when infecting with a lower number of factors to use the same amount of viral supernatant in each experiment • Add $5 \mu \mathrm{g} / \mathrm{ml}$ final concentration protamine sulphate $\cdot$ Infect in triplicate Example for infection of OSKM+ TFIID-12: • Combine $250 \mu$ viral supernatant of each of the OSKM reprogramming factors oct4, sox2, klf4, c-myc • Add $250 \mu$ of each of the TFIID-12 factors $\backslash(T B P, T A F 3,4,4 b, 5,6,7,9,10$, $11,12,13) \cdot$ Total volume in this case is $4 \mathrm{ml}$, mix gently before adding protamine sulfate $\cdot$ Add 1:1000 from a $5 \mathrm{mg} / \mathrm{ml}$ stock solution of protamine sulphate \(Sigma P4020) o Replace the medium of OG2 
MEFs with the infection mix. Use up to $4 \mathrm{ml}$ infection mix per 1 well of a 6 wells plate. Day 5 - repeat the infection once more as described in day 4 using new viral supernatant that was formed in last 24 hrs. discard the 293T cells Note: the infected MEFs should clearly have grown with a confluency of $\sim 25 \%$. If this is not the case, the quality of the batch of MEFs used may not be optimal Day 6 - change the medium of the infected OG2 MEFs to ES medium. From now on, refresh each day with ES medium Note: the infected MEFs should clearly have grown with a confluency of 50-70\% Day 7 Note: the infected MEFs should clearly have grown with a confluency of 80\% Day 8 Note: the infected MEFs should clearly have grown with a confluency of $~ 90-95 \%$ - trypsinize infected MEFs and plate all cells from $1 \times 3.5 \mathrm{~cm}$ dish to $1 \times 10 \mathrm{~cm}$ dish that has been gelatinized. Note: this is important because the MEFs will proliferate and overgrow the plate. Without proper splitting, iPS cells will only appear at the edges of the plate and the results will not be reliable. Day 20 - count iPS colonies based on morphology and GFP fluorescence. Medium composition: MEF medium - 500 ml DMEM high glucose \(Lonza BE 12-604F) - 55 ml FBS \ (Hyclone cat SV30160.03) - $5 \mathrm{ml}$ Penicillin/Streptomycin \(Lonza cat 17-603E) - $5 \mathrm{ml}$ glutamine $\backslash($ Lonza cat 17-605E) ES cell medium - 500 ml DMEM high glucose \(Lonza BE 12-604F) - 90 ml FBS \(Hyclone cat SV30160.03) - 6 ml Penicillin/Streptomycin \(Lonza cat 17-603E) - 6 ml Glutamine \(Lonza cat 17605E) - $6 \mathrm{ml}$ sodium pyruvate $\backslash($ Lonza BE 13-115E) - $6 \mathrm{ml}$ beta-mercaptoethanol $\backslash($ stock: 70 ul of beta mercapotethanol \(Sigma M-6250) in $100 \mathrm{ml} \mathrm{H2O}$, aliquot and store -20C) - $6 \mathrm{ml}$ non essential amino acids \(lonza 13-114E) - 60 ul LIF \(500 U/ml final) \(Gibco 13275-029)

\section{Timing}

20 days

\section{Anticipated Results}

Inclusion of TFIID-12 or TAF4 during OSKM-mediated reprogramming should result in a strong stimulation of the amount of iPS colonies and in the number of GFP+ cells $\backslash$ (when OG2-MEFs were used). Both intermediate and strong GFP+ cells will be formed. The intermediate GFP+ cells represents a population of which many $\backslash(\sim$ half $)$ will make it to the fully reprogrammed state upon prolonged culture.

\section{References}

1. Pijnappel W.W., Esch D., Baltissen M.P., Wu G., Mischerikow N., Bergsma A.J., van der Wal E., Han D.W., Bruch H.V., Moritz S., Lijnzaad P., Altelaar A.F., Sameith K., Zaehres H., Heck A.J., Holstege F.C., Schöler H.R. \& Timmers H.T. A central role for TFIID in the pluripotent transcription circuitry._Nature_. 2013 Mar 17. doi: 10.1038/nature11970. \[Epub ahead of print]

\section{Acknowledgements}

We thank Hermann vom Bruch for technical assistance 